\title{
Una Propuesta de Estudio de la Pragmática en el Grado de Educación Primaria
}

\author{
Elisabeth Melguizo Moreno \\ Universidad de Granada
}

Recibido: 09 marzo 2017 / Aceptado: 21 julio 2017

ISSN: $1697-7467$

\begin{abstract}
RESUMEN: En este artículo se pretende ofrecer una propuesta didáctica para el estudio de la Pragmática, una disciplina compleja y de difícil comprensión. Tomando como base la selección de una serie de actividades orales y/o escritas de carácter pragmático, presentes en distintos libros de texto de lengua castellana y literatura de Educación Primaria, se propone un análisis específico para que futuros maestros en formación puedan hacer que sus alumnos desarrollen la competencia pragmática en el aula. Los resultados evidenciaron la destreza de los universitarios para identificar esta disciplina, tras una enseñanza teórica previa de sus componentes esenciales.
\end{abstract}

Palabras clave: Pragmática, Educación Primaria, docentes, contexto, intención comunicativa.

\section{A proposal for the study of pragmatics in the Primary Education Degree}

\begin{abstract}
In this article we intend to offer a didactic proposal for the study of pragmatics, a complex and difficult to understand discipline. Taking as a basis the selection of a series of oral and/or written activities of pragmatic character, present in different Spanish language and literature textbooks of Primary Education, a specific analysis is proposed so that future teachers in training can make their students develop the competence in this field, after a previous theoretical teaching of its essential components.
\end{abstract}

Keywords: Pragmatics, Primary Education, teachers, context, communicative intention.

\section{INTRODUCCIÓN}

Cuando usamos el lenguaje diariamente tratamos de hacerlo siguiendo unas normas de corrección lingüística, pero rara vez nos percatamos del complejo mecanismo que está implícito en la transmisión de un mensaje. Comunicamos distintos hechos, pero cuando lo hacemos no solo informamos sobre algo, sino que también e indirectamente debemos interpretar la intención del hablante. No importa tanto lo que se manifiesta abiertamente para notificar un acontecimiento como lo que realmente no se expresa pero también se comunica. En este campo nos adentramos en la dimensión pragmática.

La Pragmática es una disciplina que se ocupa del significado de las palabras y las oraciones en su relación con hablantes y contextos en actos de comunicación concretos (Reyes, 1995), pero también se interesa por analizar las estrategias utilizadas en las actuaciones lingüísticas para lograr el éxito deseado (Fernández Pérez, 1996). 
La verdadera esencia de la Pragmática parece estar muy dispersa, ya que abarca aspectos muy distintos. Escandell (2006: 15-16) se pronuncia en este sentido, y entiende por Pragmática "el estudio de los principios que regulan el uso el lenguaje en la comunicación, es decir, las condiciones que determinan tanto el empleo de un enunciado concreto por parte de un hablante concreto en una situación comunicativa concreta, como su interpretación por parte del destinatario". Se puede afirmar, por tanto, que la Pragmática considera distintos factores extralingüísticos que influyen en la interpretación de los enunciados: emisor, destinatario, intención comunicativa, contexto, situación o conocimiento del mundo, etc.

Sin duda, "hablar es actuar con la palabra" (Escavy, 2009). De ahí que sean de suma importancia para la Pragmática los denominados actos de habla. Austin (1962) señaló que cuando hablamos emitimos actos (locutivos, elocutivos y perlocutivos) porque el lenguaje es ante todo acción. Los interlocutores durante el acto de comunicación intercambian información, que puede o no ser compartida por ambos. De ahí la existencia de una parcela de información pragmática de dimensiones variables (Escandell, 2006). Esta se compone de un conjunto de conocimientos, supuestos, creencias, opiniones y sentimientos de un individuo en un momento dado de la interacción verbal (Dik, 1989).

Pero, ¿cómo es posible que no coincida en algunos casos lo que decimos con lo que realmente queremos expresar? Precisamente, porque se habla de dos tipos de significados claramente definidos: el significado convencional, que es el significado literal, el compartido por todos los miembros de la comunidad lingüística; y el significado del hablante, que es el significado intencional, que depende de las circunstancias en el acto de habla. Este último es el propio de la disciplina Pragmática, ya que requiere de una interpretación para ser comprensible.

Si profundizamos un poco más en el terreno pragmático, nos daremos cuenta de que en el seno de lo implícito residen las inferencias. Cuando un emisor cualquiera emite un mensaje, su interpretación requiere de todo un proceso de descodificación. Lo que normalmente inferimos es aquello que se nos quiere decir, pero que no se dice explícitamente. Se han llegado a considerar las inferencias como "el núcleo de la comprensión e interpretación de la realidad y, por tanto, uno de los pilares de la cognición humana" (León, 2003: 23).

Sobre inferencias se pronuncia Ripoll (2015), estableciendo una clasificación de las mismas basada en una denominación genérica, y usando como criterio de clasificación las preguntas que originarían los distintos tipos de inferencias. Por ejemplo, habla de inferencias de tipo I, que responden a preguntas como: ¿a qué (o a quién) se refiere?, ¿de qué (de quién) habla el texto? De entre ellas, nos interesa fundamentalmente señalar las pragmáticas, que tienen cabida en el ámbito educativo, relacionadas con la lengua o la mejora de la comprensión lectora (Ripoll y Aguado, 2014) y aquellas relacionadas con la capacidad del estudiante de inferir el significado adecuado de expresiones irónicas (Banasik, 2013) o la moraleja de un relato (Duque y Vera, 2010; Narvaez, Gleason y Mitchell, 2010).

A tenor de lo expuesto, y teniendo en cuenta que los estudiantes de magisterio desconocen qué es la Pragmática y cuáles son sus aspectos constituyentes, se realiza un estudio en el que se da a conocer el estado de la cuestión en un grupo de 65 alumnos del Grado de maestro de Educación Primaria. 
Elisabeth Melguizo Moreno Una Propuesta de Estudio de la Pragmática en el Grado de Educación...

\subsection{Hacia una didáctica de la Pragmática: su aplicabilidad en el aula}

Hacia 1967 Chomsky introdujo los conceptos de competencia y actuación lingüística con la intención de desarrollar la competencia comunicativa del hablante. En la enseñanza de lenguas se advierte de forma clara que un aprendiz de un curso de español no concluye con éxito su aprendizaje si no consigue usar determinadas funciones comunicativas de la lengua (González, 2005). No basta con que un estudiante conozca perfectamente la gramática de un idioma, sino que sepa comunicar distintas nociones en un contexto adecuado.

Se plantea así la necesidad de "enseñabilidad" de la competencia pragmática (Kasper, 1997), y se proponen, según los autores, fórmulas pedagógicas para la enseñanza de la competencia comunicativa que permitan reconocer e identificar esta disciplina por medio de la práctica.

En el área de lengua castellana y literatura, Rodríguez (2015) señala algunos aspectos para tratar contenidos pragmáticos: trabajar con material auténtico (películas, grabaciones reales, etc.), contextualizar las muestras de habla en el espacio y en el tiempo, reflexionar sobre el uso de las fórmulas elegidas y seleccionar las expresiones de los actos de habla más rentables por su frecuencia de uso.

A hablar se aprende hablando. Sin embargo, en las aulas de primaria y secundaria los alumnos escuchan más que hablan, y este hecho hace que su competencia comunicativa sea escasa o reducida (Garrán, 1999). Es necesario, por tanto, que los docentes fomenten la capacidad comunicativa de los estudiantes, de acuerdo con varios principios (Fernández, 1994): a) el de no sustitución: no enseñar lo que los estudiantes ya saben, sino solo llevar a cabo acciones docentes operativas; b) el de anticipación: adaptando nuestra intervención a las necesidades de los alumnos, con actividades que les permitan pensar y no actuar de forma repetitiva; c) el de motivación: plantear tareas de acuerdo a sus intereses; d) el de socialidad: considerar lo que el alumno es capaz de aprender solo y con ayuda; e) el de diversidad metodológica: acompañada de diversidad de recursos (textos grabados, chistes, etc.); f) el de reciprocidad entre teoría y práctica: fusión; g) sistematicidad intradidáctica: estrecha relación entre objetivos y contenidos, con metodología y criterios de evaluación; $\mathrm{y}, \mathrm{h})$ principio de adecuación epistemológica: conversión de contenidos científicos en contenidos curriculares.

Garrán (1999) y Rius (1987) señalan que muchos de los contenidos curriculares de primaria y secundaria referentes a la lengua oral pueden ser estudiados desde un enfoque comunicativo a partir de contenidos pragmáticos. Se trata, por tanto, de fomentarlos considerando:

a) Los aspectos relacionados con la estructura de la lengua: a qué obedece que el hablante haya elegido un orden de presentación de las palabras al construir su enunciado o cómo los deícticos nos permiten saber quién es el emisor, el destinatario o las circunstancias de expresión.

b) Los actos de habla, que permiten trabajar contenidos como las intenciones comunicativas relacionadas con la expresión de sentimientos, narrar, describir, etc., e incluso ofrecer formas adecuadas a las distintas situaciones y propósitos comunicativos explícitos en estructuras textuales.

c) Los principios de cooperación y máximas de Grice, a través de textos literarios de la tradición oral (canciones, romances, etc.), textos de los medios de comunicación social, grabaciones, conversaciones, etc. 
Por otro lado, la implicatura conversacional permite desarrollar la interpretación de mensajes no explícitos en los textos orales (doble sentido, sentido humorístico) o, incluso, la observación de capacidades expresivas de la lengua a partir del análisis de textos de la lengua oral.

En definitiva, se puede concluir diciendo que "la enseñanza/aprendizaje de la lengua oral en las aulas de primaria y secundaria puede plantearse desde una perspectiva pragmática, puesto que el desarrollo de sus contenidos nos permite una visión general y amplia del hecho comunicativo" (Garrán, 1999:120).

\subsection{Tipos de texto: análisis de sus componentes pragmáticos}

En el contexto del aula, como se detalla más adelante, se requirió al alumnado la búsqueda de componentes pragmáticos en distintos manuales de lengua castellana y literatura, para conocer sus habilidades en los siguientes tipos de texto: refranes, frases hechas, adivinanzas y chistes.

a) Refranes. El refrán es "una sentencia lingüística concreta, realizada por un hablante en una situación comunicativa; se define dentro de una teoría pragmática, y su interpretación depende de su contenido semántico pero especialmente de las condiciones contextuales de emisión" (Ramírez y Álvarez, 2006: 223). Por este motivo, los autores consideran el refrán como objeto de estudio de la pragmadialéctica, puesto que en él se reconocen fenómenos de inferencia, procesos de cooperación desarrollados por Grice (1975) para su comprensión o la identificación de elementos que otorgan relevancia (Sperber y Wilson, 1986).

b) Frases hechas. García-Page (2015) señala los valores culturales y simbólicos de las unidades fraseológicas, así como su función pragmática y textual. Las locuciones $\mathrm{y}$, sobre todo, las fórmulas rutinarias, se han tratado fundamentalmente en el nivel pragmático y textual, y transportan información acerca de la cultura de un pueblo o nación, transmitiendo sus usos, costumbres, supersticiones, etc.

c) Adivinanzas. Adivinar un objeto, una persona o un animal es un ejercicio intelectual que requiere interpretar, deducir, extrapolar información implícita, hacer inferencias, etc. El juego, el ingenio, lo lúdico y el efecto sorpresa son indispensables (Beltrán, 2013).

d) Chistes. Los chistes juegan con la ironía, el sarcasmo, el doble sentido de las palabras, lo implícito, etc. Se puede decir que se trata de un subgénero humorístico y pseudoliterario que se mueve en el terreno de la ficción y se caracteriza por su función lúdica, intencionalidad cómica, brevedad, efecto-sorpresa y cierre previsto (Vigara, 2013).

\section{Hipótesis y ObJetivos}

La hipótesis de este trabajo se sustenta en el hecho de que los alumnos que actualmente cursan primero del Grado de maestro de Educación Primaria en la Universidad de Granada no saben con exactitud qué es la Pragmática y cuáles son sus componentes principales. 
Elisabeth Melguizo Moreno Una Propuesta de Estudio de la Pragmática en el Grado de Educación...

Desconocen, por tanto, su aplicabilidad a la enseñanza, y les resulta imposible identificar, en primera instancia, los elementos constituyentes de la disciplina en actividades insertas en manuales de lengua castellana y literatura de esta etapa educativa. Por ejemplo, en una práctica un grupo de estudiantes seleccionó una actividad de expresión oral, que en su opinión era pragmática, y consistía en que los alumnos se presentaran ante un compañero siguiendo un guion y utilizando un vocabulario dado en una ficha del manual. Algunas preguntas del ejercicio eran: ¿cómo te llamas?, ¿cuántos años tienes?, etc. Se trata de una actividad en la que resulta imposible que quede reflejada una intencionalidad comunicativa en las respuestas de los hablantes, ya que las cuestiones que se plantean son literales y se responderán exclusivamente con respuestas explícitas.

Por esta razón, se plantea la necesidad de realizar una explicación teórica previa de estos contenidos y, posteriormente, diseñar una propuesta pragmática, con el fin de verificar esta enseñanza.

Por lo que a los objetivos se refiere, es necesario distinguir entre el objetivo general de la experiencia llevaba a cabo y los objetivos específicos que se derivan de este. Como se ha mencionado, este artículo pretende, en líneas generales, dar a conocer a los futuros maestros en formación una propuesta didáctica de estudio de la Pragmática. Teniendo en cuenta este propósito, se formulan distintos objetivos específicos. A partir de las actividades basadas en refranes, frases hechas, adivinanzas y chistes, los estudiantes universitarios deben:

a) Identificar los componentes pragmáticos esenciales en actividades incorporadas en libros de texto de lengua castellana y literatura en varios cursos de Educación Primaria, considerando la propuesta del docente.

b) Reconocer en los libros de texto las actividades de lengua oral y/o escrita que mejor reflejan la disciplina Pragmática a través del análisis de los distintos tipos de textos seleccionados.

c) Realizar una adecuada transposición didáctica de los contenidos pragmáticos asimilados mediante un análisis contrastivo entre el profesorado de diferentes centros.

\section{Metodología}

La metodología llevada a cabo en esta experiencia práctica ha sido el denominado análisis documental. Fox (2005) se refiere a este método como el tratamiento de textos que tiene como finalidad elaborar una nueva representación de su contenido para hacerlo más asequible y comprensible.

Para la selección de documentos se ha utilizado una parrilla de doble entrada donde se han considerado solamente los trabajos más representativos y relevantes redactados por los estudiantes de acuerdo con los objetivos planteados por el docente, es decir, aquellos que mostraban una identificación total de los componentes pragmáticos explicados por el profesor en las sesiones teóricas y que se centraban en las tipologías textuales seleccionadas (refranes, frases hechas, etc.).

El docente llevó a cabo una enseñanza teórica previa que tuvo lugar en 2 sesiones de clase, con una duración de 4 horas en total. Se explicó el concepto de pragmática, los componentes esenciales y su aplicación a la enseñanza de la lengua. 


\subsection{Participantes}

En este estudio participaron 65 estudiantes que cursan la asignatura Didáctica de la lengua española I ( $1^{\circ}$ curso) del Grado de Educación Primaria de la Facultad de Ciencias de la Educación de la Universidad de Granada. Se procedió a una selección de 30 textos pertenecientes a la tipología de trabajos académicos que los alumnos realizaron en grupos durante los seminarios prácticos. Se trataba de trabajos grupales de una extensión no superior a 10 páginas.

\subsection{Obtención y análisis de datos}

Los datos se han extraído de los trabajos resultantes de una práctica en la que los estudiantes debían realizar la siguiente tarea: localizad en distintos manuales de las editoriales Santillana, Anaya y Vicens Vives de lengua castellana y literatura de la etapa de Educación Primaria los aspectos pragmáticos explicados previamente por el docente. Prestad atención fundamentalmente a los apartados dedicados a la lengua oral y redactad un breve informe donde analicéis y valoréis dichos aspectos.

La propuesta que se plantea más abajo se fundamenta en el análisis de los siguientes elementos de la Pragmática y se centra en las tipologías textuales referidas ${ }^{1}$ :

a. Enunciado. Es el resultado del acto de enunciación por parte del emisor. Su extensión es variable, estando delimitada por el cambio de emisor o enunciador. Es la unidad de análisis de la Pragmática.

$i$. Sobre el enunciado los estudiantes tienen que aclarar en cada una de las actividades si es de fácil comprensión por el alumno, claro y conciso; o si, por el contrario, es incomprensible o de difícil interpretación.

b. La intención comunicativa. Es el propósito o meta que el emisor quiere conseguir por medio de su enunciado.

$i$. Los universitarios deben decir si hay una intención clara en el enunciado $\mathrm{y}$, por tanto, si los niños podrán realizar la actividad sin ningún problema, sabiendo el propósito desde el principio.

c. Ámbitos de uso de la lengua ${ }^{2}$. Cada acto de uso de la lengua se inserta en una serie de ámbitos en que se organiza la vida social; es decir, la producción y recepción de enunciados responden a unas áreas de interés o se insertan en unas esferas de acción en las cuales el individuo debe ser competente comunicativamente. El

\footnotetext{
${ }^{1}$ Se realizará una breve explicación de cada uno de los aspectos pragmáticos trabajados en las actividades con el fin de delimitar los objetivos concretos que cada estudiante debía alcanzar con la propuesta planteada.

${ }^{2}$ En los apartados $c$. (Ámbitos de uso de la lengua), $d$. (Contexto), e. (Situación), $f$. (Marco del discurso) y $g$. (Aportaciones de la Pragmática a la enseñanza de la lengua) los estudiantes tienen que identificar qué elementos de los mencionados reconocen en las actividades localizadas en los manuales seleccionados de Educación Primaria y explicar cómo se presentan.
} 
Elisabeth Melguizo Moreno Una Propuesta de Estudio de la Pragmática en el Grado de Educación...

Marco Europeo de Referencia para las lenguas establece los siguientes ámbitos:

i. Ámbito personal, que es el de la vida privada del individuo (familia y amigos).

ii. Ámbito público, que es el de actuación de la persona como miembro de la sociedad. En él se realizan transacciones de distinto tipo y con intenciones variadas (sociales, administrativas, etc.)

iii. Ámbito profesional, en el que la persona desarrolla su profesión.

iv. Ámbito educativo, en el que el individuo participa de manera organizada del aprendizaje, sobre todo en el seno de una institución educativa.

d. Contexto. Es el conjunto de factores extralingüísticos que condicionan tanto la producción de un enunciado como su significado. Se distinguen varios tipos de contexto:

i. Contexto discursivo o entorno lingüístico, integrado por los elementos que constituyen el enunciado. Se conoce también como cotexto.

ii. Contexto circunstancial, espacio y tiempo que condicionan el enunciado.

iii. Contexto social, que incluye elementos culturales (rituales) que acompañan a la enunciación. Ligado a este tipo de contexto, se encuentra el denominado conocimiento del mundo, esto es, los marcos conceptuales o de referencia que pertenecen a la experiencia previa de la realidad de los interlocutores que pueden compartir o no, o en diversos grados.

e. Situación. Según el MCER, las situaciones surgen en los ámbitos señalados y dependen de factores como el lugar y el momento, las instituciones, las personas implicadas, las acciones, etc. Dependiendo de estos factores, se puede hablar de situaciones que requieren el uso oral o el uso escrito.

f. Marco del discurso ("tipo de discurso en el cual queda comprendido el acto comunicativo'). Entre las modalidades orales destacan la conversación, la discusión, el debate, la entrevista, etc. En las modalidades escritas: la correspondencia (privada o comercial), el currículum vitae (comercial o académico), escritos académicos (trabajo monográfico ficha...), etc.

g. Aportaciones de la Pragmática a la enseñanza de la lengua:

i. Atender a ámbitos de uso y a intenciones comunicativas reales o verosímiles. El enfoque por tareas potencia la enseñanza-aprendizaje de la lengua como un medio para alcanzar un fin.

ii. Abordar la enseñanza y el aprendizaje de la lengua atendiendo a la variedad discursiva, en función de las variables de ámbito, contextuales y situacionales:

a. Atención a las tipologías textuales (narración, descripción, argumentación, exposición, diálogo). En el aula se ha de trabajar con textos completos y auténticos (no creados exclusivamente para la educación o manipulados). 
b. Atención a la lengua oral, resultado de trabajar sobre una lengua real y contextualizada.

iii. Potenciación del estilo interactivo en el aula:

Búsqueda de estrategias comunicativas:

1. Planificación y modificación del entorno (distribución de espacios y materiales).

2. Alternancia de trabajos individuales, por parejas o pequeño grupo.

3. Creación de rutinas interactivas (saludos, introducción-conclusión de tareas, despedida...)

4. Búsqueda de estrategias de intervención lingüística

a. Gestión de la conversación (presentaciones, debates, exposiciones...)

b. Uso de estrategias educativas implícitas (feed-back, ampliaciones...)

c. Ajuste de características formales (diversidad léxica, complejidad sintáctica).

iv. Desarrollo de las cuatro habilidades básicas de la comunicación (Cassany, Luna y Sanz, 2001):

a. Expresión oral (hablar): dramas, escenificaciones, simulaciones, lectura en voz alta, etc.

b. Comprensión oral (escuchar): juegos mnemotécnicos, completar cuadros, transferir información, etc.

c. Comprensión escrita (leer): ejercicios de desarrollo de la percepción, la memoria, la anticipación, relleno de espacios, etc.

d. Expresión escrita (escribir): redacciones, dictados, comentarios de textos, trabajo por tareas, etc.

\section{Resultados}

La localización de los componentes pragmáticos aludidos en distintas actividades de libros de texto de Educación Primaria no fue tarea fácil para los universitarios, ya que primero tuvieron que comprender perfectamente el concepto y objeto de la Pragmática y, posteriormente, reconocer sus componentes esenciales en la práctica.

Los maestros en formación se centraron básicamente en aquellos ejercicios correspondientes al ámbito oral de la lengua, que es donde mayoritariamente quedaba reflejaba la disciplina, y a las tipologías textuales requeridas, aunque muchos de ellos también identificaron la Pragmática en actividades escritas.

A continuación se muestran los resultados del análisis realizado por los estudiantes universitarios que evidencia su aprendizaje de la Pragmática. En él se puede comprobar que no solamente fueron capaces de detectar actividades pragmáticas centradas en las tipologías textuales requeridas, sino que también las explicaron con destreza, tras la propuesta que le plantea el docente. 
Elisabeth Melguizo Moreno Una Propuesta de Estudio de la Pragmática en el Grado de Educación...

\subsection{Refranes}

La actividad que los alumnos seleccionaron para trabajar la Pragmática fue la número 1 (Véase figura 1), "perteneciente a $4 .^{\circ} \mathrm{EP}^{3}$ de la editorial Santillana”. En ella, los niños debían sustituir los dibujos por palabras y escribir refranes. Los elementos pragmáticos detectados por los universitarios fueron los siguientes:

- Enunciado. Es fácil y acorde al vocabulario para los alumnos del segundo ciclo. No obstante, aclaran que el profesor debe explicar previamente qué es un refrán y cómo interpretar su significado.

- Ámbitos de uso de la lengua: educativo (el alumno está inmerso en la institución escolar) y público (los refranes son sentencias breves, cuyo uso es compartido por una comunidad y promueve la reflexión, transmitiendo una enseñanza o sirviendo como ejemplo). Los alumnos de EP manifiestan que el significado que se extrae de los refranes siempre implica un consejo o lección para aplicar posteriormente en la vida.

- Contexto: discursivo (el refrán se enclava en un proceso de comparación, que casi siempre desemboca en un proceso de metaforización. Establece una situación tipificada); social (los refranes son parte de la cultura popular; una realidad viva, dinámica, social y actual, de la cual todas las personas, de forma consciente e inconsciente, y en algún momento y grado, participan).

- Situación: requiere un uso escrito.

- Marco del discurso. Modalidad escrita.

- Aportaciones de la Pragmática: atiende a ámbitos de uso y a intenciones comunicativas reales o verosímiles; se potencia igualmente la enseñanza y aprendizaje de la lengua como un medio para alcanzar un fin; exposición de los refranes; la comprensión escrita a través de técnicas de pregunta directa, comparación de textos, etc.

\subsection{Frases hechas}

El libro consultado en esta ocasión por los futuros maestros pertenecía al tercer curso de la editorial Anaya. Concretamente, eligieron la actividad número 1 (Véase figura 2) y explicaron de la siguiente forma los componentes pragmáticos:

Figura 2. Actividad 1. Editorial Anaya

\footnotetext{
${ }^{3}$ Se usarán las siglas EP para referirse a la etapa de Educación Primaria que los estudiantes están cursando en la
} Universidad 
- Enunciado: Sobre él dijeron que se trataba de un enunciado de fácil comprensión por los niños y que no requería ninguna explicación.

1 Copia y relaciona, en tu cuaderno, cada frase hecha con su significado:

- Hace las cosas deprisa y corriendo.

- Esta sala es un horno. - Es fuerte como un toro.
- Hace mucho calor.

- Tiene mucha fuerza.

- Hace las cosas sin poner atención.

- Ámbitos de uso de la lengua: educativo, ya que se corresponde con un libro de lengua de Educación Primaria, donde el alumno participa de forma organizada en el aprendizaje; personal, puesto que la elocución de las frases hechas requiere un contexto en el que participen familia y/o amigos; público, por nuestra actuación como miembros de la sociedad y profesional, ya que se entiende que dichas frases se pueden decir en un contexto donde desarrollamos nuestra profesión (maestros y profesores).

- Contexto: se desarrolla un contexto circunstancial, determinado por el enunciado, y un contexto discursivo, pues comprende elementos como la intencionalidad del emisor.

- Situación: requiere del uso escrito, ya que los estudiantes deben escribir dichas frases en su cuaderno; aunque también, y con mucha frecuencia, se pronuncian en multitud de situaciones orales de la vida cotidiana.

- Marco del discurso: modalidad escrita académica.

- Aportaciones de la Pragmática: basada en tareas con el objetivo final de comprender el significado de las expresiones en las frases hechas del tema; atiende, además, a la lengua oral a través de expresiones o frases hechas; se puede propiciar también el estilo interactivo, y desarrolla como principal habilidad la comprensión escrita.

\subsection{Adivinanzas}

La actividad que los estudiantes de Primaria seleccionaron para explicar la Pragmática, en este caso, fue la siguiente (Véase figura 3).

- Enunciado: acorde al vocabulario de un alumno de $6 .^{\circ}$ de EP. No obstante, es conveniente que el docente realice una aclaración sobre el contenido de la actividad.

- Ámbitos de uso de la lengua. El individuo participa de forma organizada en el aprendizaje (educativo), y público, ya que el hablante actúa como miembro de una sociedad. En concreto, las adivinanzas se trasmiten de generación en generación y forman parte de nuestra cultura. Por tanto, es muy importante transferir estos conocimientos para que las adivinanzas prevalezcan en la sociedad. 
Elisabeth Melguizo Moreno Una Propuesta de Estudio de la Pragmática en el Grado de Educación...

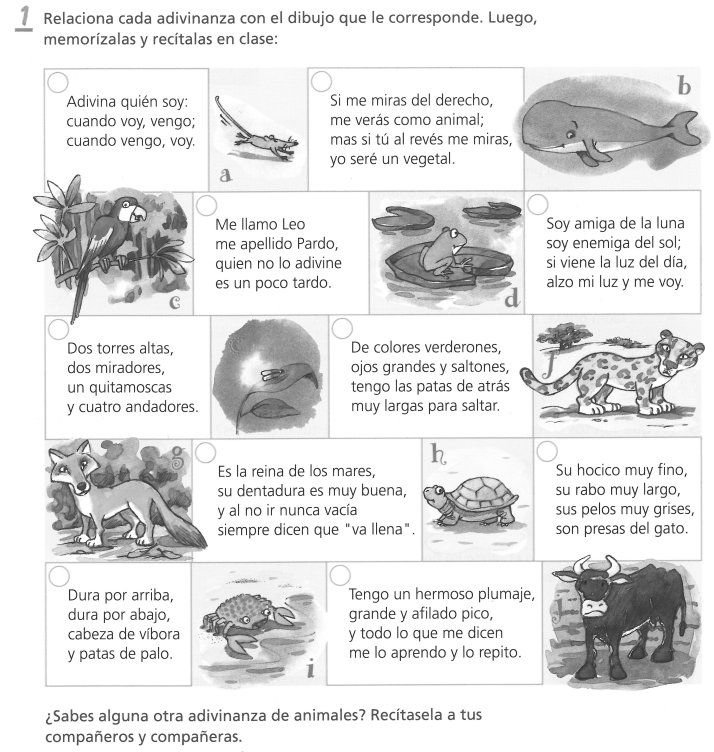

Figura 3. Actividad 1. Editorial Vicens Vives
- Contexto: es discursivo, porque se compone de todas las unidades lingüísticas y gramaticales que aparecen en cada adivinanza. Y también hay un contexto social, puesto que las adivinanzas son elementos culturales que la sociedad ha trasmitido.

- $\quad$ Situación. Es de uso escrito y oral, ya que primeramente los alumnos han de leer las adivinanzas plasmadas en el libro y posteriormente, memorizarlas y recitarlas oralmente en clase.

- Marco del discurso. Se centra en una modalidad oral puesto que, tras realizar la primera parte de la actividad (unir la adivinanza con cada dibujo), el alumno deberá recitar a sus compañeros alguna adivinanza de animales. Por tanto, se iniciará una especie de tertulia en la que el alumnado intercambiará información entre sí.

- Aportaciones de la Pragmática. Destaca la variedad discursiva (exposición de las adivinanzas), y una atención a la lengua oral, con la exposición de nuevas adivinanzas. Entre las habilidades básicas de la comunicación son fundamentales: la comprensión escrita (leer), ya que el ejercicio trata sobre el desarrollo de la memoria; y la expresión oral (recitación). Además, se fomenta el desarrollo del conocimiento a través de técnicas de localización de parejas.

\subsection{Chistes}

En esta actividad, "perteneciente a $6 .^{\circ}$ EP de la editorial Anaya" (Véase figura 4), los alumnos manifestaron que se evidencia un enunciado claro y preciso, aunque los niños pueden tener dudas en relación al significado de "chiste", por lo tanto sería conveniente que el docente aclarara su significado antes de realizar la actividad.

En relación al ámbito de uso de la lengua, destaca el ámbito educativo, ya que el estudiante aprenderá distintos significados de la palabra mancha/Mancha a través del chiste.

El contexto de la actividad es de doble tipo, según los maestros en formación: discursivo, porque hay coherencia y cohesión entre los elementos de las oraciones presentadas; y social, porque incluye elementos culturales y requiere un conocimiento del mundo. Han de ser conocedores de la región de La Mancha.

En relación a la situación, es de uso escrito porque los niños han de leer el enunciado y explicar los significados de ambas palabras en su cuaderno. 
4 Lee el chiste y explica los significados de las palabras Mancha/mancha.

Profesor señalando un mapa:

- Jaimito, ¿sabes dónde está La Mancha?

- ¡Sí, profesor!

- ¿Me puedes decir dónde?

- En la manga de su chaqueta.

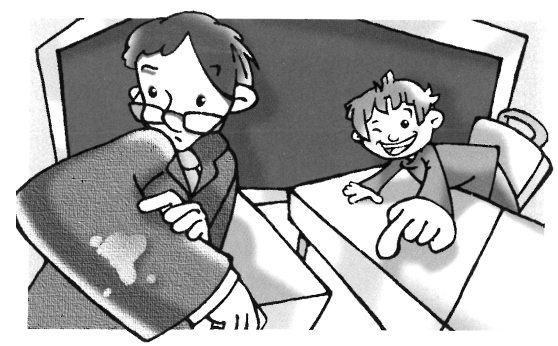

Figura 4. Actividad 4. Editorial Anaya

Por otro lado, el marco del discurso sería una modalidad escrita, el chiste, que se presenta en forma de diálogo entre dos interlocutores. En último lugar, las aportaciones de la Pragmática, destacan el uso de las siguientes destrezas: comprensión escrita, porque el estudiante debe leer el chiste para su posterior interpretación; y expresión escrita, puesto que el niño debe escribir los significados de las palabras Mancha/mancha en su cuaderno.

\section{Discusión y Conclusiones}

Con este estudio se ha pretendido poner de manifiesto los resultados obtenidos tras la puesta en marcha de una propuesta pragmática en futuros docentes en formación.

Tras la explicación previa del docente de los componentes pragmáticos, se ha procedido a la selección de 30 textos académicos realizados por los alumnos en seminarios de trabajo. Dicha selección se ha realizado en función del grado de representatividad y relevancia, en cuanto a presencia de la Pragmática en las actividades sobre las siguientes tipologías textuales: refranes, frases hechas, adivinanzas y chistes.

Como se ha podido comprobar, los alumnos han logrado alcanzar con éxito los objetivos planteados inicialmente en este trabajo. En primer lugar, han sido capaces de identificar perfectamente los componentes pragmáticos explicados previamente por el docente en las actividades mostradas. Asimismo, han reconocido las actividades de lengua oral y/o escrita que mejor plasmaban los elementos lingüísticos estudiados. Por último, se ha podido comprobar que las explicaciones dadas por los estudiantes en cada actividad evidencian su grado de conocimiento de la Pragmática, un saber que les permitirá realizar una correcta transposición de dichos contenidos cuando desarrollen en un futuro próximo su labor docente en Educación Primaria.

Sobre la aplicabilidad didáctica que tienen las tipologías textuales estudiadas, se pronuncian distintos autores. Ramírez y Álvarez (2006) se refieren al potencial didáctico de los 
refranes en clase de lengua castellana y literatura, puesto que permiten desarrollar procesos de argumentación oral o escrita y fomentar la creatividad mediante actividades como: lectura expresiva de textos que contengan refranes, formulación de preguntas sobre el contenido del refrán, comprensión de su sentido global, explicación del contexto pragmático del refrán, etc. Es el docente el que debe plantear actividades transversales para trabajar el refrán desde una perspectiva multidimensional (Alcalá y Rasero, 2001).

En relación a las frases hechas, los alumnos deben entender el significado de las mismas y, para ello, han de poseer un amplio conocimiento del mundo que les rodea. De la importancia que tiene enseñar fraseología surgió la disciplina fraseodidáctica o didáctica de la fraseología (González Rey, 2012) que arrancó de la mano de Charles Bally hacia 1909.

De igual forma, las adivinanzas constituyen un referente fundamental para desentrañar el complejo proceso que se pone en marcha para desambiguar términos, asignar un referente concreto a algo de lo que se está hablando o enriquecer la información no explícita de un texto. Ramírez Gelves (2003) revela que la adivinanza hace que el hablante tome conciencia de estos tres procesos que, en un principio, quedan diluidos por el juego del despiste o la confusión de información que propone la adivinanza. Beltrán (2013) advierte la importancia que conceden Cerrillo y Miaja, los autores de la obra que reseña, al estudio pragmático de la adivinanza. Señalan que posee potencionalidades didácticas por su capacidad de instruir implicando y deleitando al receptor más pequeño en la transmisión de conocimientos sobre el mundo de su comunidad.

Por último, los chistes pueden servir como motivación y emplearse como recurso didáctico "para ilustrar un rasgo de la lengua, para poner al alcance del alumno unos contenidos que no entiende con claridad y como un ejercicio de reflexión sobre el idioma" (García Surrallés, 1992: 57). El chiste requiere de un saber compartido por los comunicantes en una especie de acuerdo-cooperación. Este juego social de ingenio pretende divertir mediante la transmisión de un mensaje lúdico cargado de connotaciones (Vigara, 2013). Todo un proceso cognitivo que pone en marcha los esquemas mentales del sujeto para conectar la realidad del chiste con supuestos socioculturales y situaciones de la vida cotidiana (Padilla y Gironzetti, 2012).

\section{REFERENCIAS}

Alcalá, J. y Rasero, J. (2001). "El refrán como herramienta didáctica en Educación Primaria”, en E. Menéndez Ayuso y A. Delgado Cabrera (coords.), Lengua y cultura: enfoques didácticos. Universidad de las Palmas de Gran Canaria: Servicio de Publicaciones, 147-164.

Austin, J. L. (1962). How to do things with words. Nueva York: Oxford University Press.

Banasik, N. (2013). "Non-literal speech comprehension in preschool children -An example from a study on verbal irony", en Pyshcology of Language and Communication, 17, 3: 309-323.

Beltrán, R. (2013). “Sobre 'zazaniles' y 'quisicosas': estudio del género de la adivinanza”. Reseña de Pedro C. Cerrillo y M ${ }^{a}$ Teresa Miaja, Cuenca: Universidad de Castilla-La Mancha, 2011, Boletín de Literatura Oral, 3: 167-170.

Cassany, D., Luna, M. y Sanz, G. (2001). Enseñar lengua. Barcelona: Graó.

Dik, S. C. (1989). The Theory of Functional Grammar. Dordrecht: Foris.

Duque, C. P. y Vera, A. V. (2010). "Exploración de la comprensión inferencial de textos narrativos en niños de preescolar", en Revista Colombiana de Psicología, 19, 1: 21-35, available from: http://dialnet.unirioja.es/descarga/articulo/3641261.pdf, accessed 04 January, 2017. 
Escandell, M. V. (2006). Introducción a la Pragmática. Barcelona: Ariel.

Escavy, R. (2009). Pragmática y textualidad. Murcia: Universidad de Murcia.

Fernández Pérez, M. (1996). "El campo de la lingüística aplicada. Introducción”, en M. Fernández Pérez (coord.), Avances en Lingüistica Aplicada. Santiago de Compostela: Servicio de Publicaciones, 11-46.

Fernández, M. (1994). Las tareas de la profesión de enseñar. Madrid: Siglo XXI.

Fox, V. (2005). Análisis documental de contenido: principios y prácticas. Buenos Aires: Alfagrama.

García Surrallés, C. (1992). "El chiste como recurso en la didáctica de la primera lengua", en El Guiniguada, 3,1 (Ejemplar dedicado a: Actas del II Congreso Internacional de la Sociedad de Didáctica de la lengua y la literatura), Las Palmas de Gran Canaria, 57-64.

García-Page, M. (2015). "Aspectos culturales y pragmáticos de los fraseologismos. Estado de la cuestión y pautas para su estudio", en I. Carrasco Cantos y S. Robles Ávila (coords.), Pragmática, discurso y norma. Madrid: Arco-Libros, 235-263.

Garrán, M. L. (1999). "Desarrollo de la lengua oral en el aula: una visión Pragmática", en Lenguaje y textos, 13: 107-120, available from: http://ruc.udc.es/dspace/bitstream/handle/2183/8071/ LYT_13_1999_art_7.pdf?sequence=1, accessed 27 December, 2016.

González Rey, M I. (2012). "De la didáctica de la fraseología a la fraseodidáctica", en Paremia, 21: $67-84$

González, A. (2005). "La didáctica de la pragmática en el proceso de enseñanza-aprendizaje de lenguas segundas y extranjeras. Algunas consideraciones", en Lenguaje y textos, 23: 127-138, available from: http://ruc.udc.es/dspace/bitstream/handle/2183/8234/LYT_23_2005_art_10. pdf? sequence=1, accessed from 03 January, 2017.

Grice, P. (1975). "Logic and y conversation", en P. Cole y J. L. Morgan (eds.), Sintax and semantics vol 3: speech acts. Nueva York: Academic Press, 43-58.

Kasper, G. (1997). "Can Pragmatic Competence be taught?". Honolulu: University of Hawaii, en Second Language Teaching and Curriculum Center, available from: http://www.nflrc. hawaii.edu/NetWorks/NW06/, accessed 03 January, 2017.

León, J. A. (2003). "Una introducción a los procesos de inferencias en la comprensión del discurso escrito", en J. A. León (ed.), Conocimiento y discurso. Claves para inferir y comprender. Madrid: Pirámide, 23-43.

Narvaez, D., T. Gleason y Mitchell, C. (2010). "Moral virtue and practical wisdom: theme comprehension in children, youth and adults". Journal of Genetic Psychology, 171, 4: 363-388, available from: http://www3.nd.edu/ dnarvaez/documents/NarvaezGleasonGenPsy2010.pdf, accessed 10 December, 2016.

Padilla, X. A. y Gironzetti, E. (2012). "Humor e ironía en las viñetas cómicas periodísticas en español e italiano: un estudio pragmático y sociocultural”, en Foro hispánico: revista hispánica de Flandes y Holanda, 44: 93-133.

Ramírez Gelves, S. (2003). "La partícula ‘eh’ y la Teoría de la Relevancia. Un ejemplo de contenido procedimental", en Estudios filológicos, 38: 157-177.

Ramírez, R. y Álvarez, T. (2006). “Aproximación a un análisis pragmático del refrán: dimensión argumentativa”, en Didáctica (Lengua y Literatura), 18: 221-236.

Reyes, G. (1995). El abecé de la Pragmática. Madrid: Arco-Libros.

Ripoll, J. C. (2015). "Una clasificación de las inferencias pragmáticas orientada a la didáctica", en Investigaciones sobre Lectura, 4: 107-122.

Ripoll, J. C. y Aguado, G. (2014). "La mejora de la comprensión lectora en español: un metaanálisis", en Revista de Psicodidáctica, 19, 1: 27-44, available from: DOI: 10.1387/ RevPsicodidact.9001, accessed 12 December, 2016. 
Elisabeth Melguizo Moreno Una Propuesta de Estudio de la Pragmática en el Grado de Educación...

Rius, M. D. (1987). Lenguaje oral. Proyecto de metodología cientifica para el desarrollo de la comunicación en la escuela. Madrid: Seco Olea.

Rodríguez, A. (2015). "La competencia Pragmática en el alumno brasileño de ELE", en Ministerio de Educación, Cultura y Deporte (ed.), Actas del XXIII seminario de dificultades especificas de la enseñanza del español a lusohablantes: la cultura en la enseñanza del español a brasileños. Brasilia, DF: Consejería de Educación, 249-260.

Sperber, D. y Wilson, D. (1986). Relevance communication and cognition. Oxford: Black Well.

Vigara, A. M (2013). "Sobre el chiste, texto lúdico", en Espéculo: Revista de Estudios Literarios, 50: 8-27. 